\title{
CENTRAL APPROXIMATE UNITS IN A CERTAIN IDEAL OF $L^{1}(G)$
}

\author{
ERNST KOTZMANN AND HARALD RINDLER
}

\begin{abstract}
In this paper we show that for a locally compact group $G$ the ideal $L^{0}(G)=\left\{f \mid f \in L^{1}(G), \int f=0\right\}$ of $L^{1}(G)$ has multiple approximate units belonging to the center of $L^{0}(G)$ iff $G$ has a basis of invariant neighbourhoods of 1 and if all conjugacy classes of $G$ are precompact, or, equivalently, the group of inner automorphisms is precompact in the group of all topological automorphisms. In a sense this is part of the problem to characterize certain classes of groups by properties of the group algebra.
\end{abstract}

Analogously to the papers of Reiter [7] and Mosak [5] we will characterize the class $[F I A]^{-}$by the existence of approximate units in a certain ideal of the group algebra. The class $[F I A]^{-}$consists of all groups such that the group of inner automorphisms is precompact in the group of all continuous automorphisms (see $\left[1\right.$, p. 2]). It is known that $[F I A]^{-}=[F C]^{-} \cap[S I N]$, where $[S I N]$ is the class of all groups having a basis of neighbourhoods of 1 invariant under all inner automorphisms, and $[F C]^{-}$is the class consisting of all groups such that every conjugacy class is precompact (see [2, p. 325, Theorem 4.1]).

In [8] Reiter shows the following theorem:

(1) A locally compact group $G$ has the property $P_{1}$ (this property is equivalent to the amenability of $G$ ) iff the ideal $L^{0}(G)$ consisting of all functions $f$ such that $f \in L^{1}(G)$ and $\int f=0$, has multiple, approximate, left units (i.e. there exists a net $\left(u_{\alpha}\right)_{\alpha \in A}$ such that $\lim _{\alpha} u_{\alpha} * f=f$ for every $\left.f \in L^{0}(G)\right)$.

In [5] Mosak characterizes the class [SIN] by:

(2) $G \in[S I N]$ iff $L^{1}(G)$ has bounded, multiple, approximate, two-sided units belonging to the center of $L^{1}(G)$.

We prove the following

THEOREM. The following statements are equivalent:

(i) $G \in[F I A]^{-}$.

(ii) $L^{0}(G)$ has multiple, approximate units belonging to the center of $L^{0}(G)$.

REMARK. Every abelian or compact and, more generally, every central group is an $[F I A]^{-}$-group.

First we prove

Received by the editors July 3, 1974.

AMS (MOS) subject classifications (1970). Primary 22D15, 22D05, 43A20.

Key words and phrases. Locally compact group, group algebra, convolution, center of an algebra, central function, approximate unit, compact invariant neighbourhood, amenable group, precompact conjugacy class.

- American Mathematical Society 1976 
Proposition. The following statements are equivalent:

(a) $G \in[F C]^{-}$.

(b) Given any $\varepsilon>0$ and any compact subset $K \subset G$, there exists a central function $s \in L^{1}(G)$ such that $s \geqslant 0, \int s=1,\left\|L_{y} s-s\right\|_{1}<\varepsilon, y \in K$, where $L_{y}$ denotes the operator on $L^{1}(G)$ defined by $L_{y} s(x)=s\left(y^{-1} x\right)$, for a.e. $x \in G$.

(c) There exists a net $\left(w_{\beta}\right)_{\beta \in B}, \int w_{\beta}=1$, such that $w_{\beta}$ belongs to the center of $L^{1}(G)$ and $\lim _{\beta} w_{\beta} * f=0$ for all $f \in L^{0}(G)$.

RemarK. If we omit the property "central" in (b), we obtain just the property $P_{1}$.

Proof. (a) $\Rightarrow$ (b). First we consider the discrete case. It follows from [3, p. 253 , Satz 1], that for every compact, invariant subset $K_{0}$ of a discrete group $G \in[F C]^{-}$the formula

$$
\varliminf_{n} m_{G}\left(K_{0}^{n+1}\right) / m_{G}\left(K_{0}^{n}\right)=1
$$

holds, where $m_{G}\left(K_{0}\right)$ denotes the Haar measure of the set $K_{0}$. It follows that for every $\varepsilon>0$ there exists an integer $n \in \mathbf{N}$ such that

$$
m_{G}\left(K_{0}^{n+1} \backslash K_{0}^{n}\right) m_{G}\left(K_{0}^{n}\right)^{-1}<\varepsilon
$$

(we may assume that $K_{0}^{n} \subset K_{0}^{n+1}$ ). Now let $K$ by any compact subset and $\varepsilon>0$; there exists an invariant compact set $K_{0}$ such that $K_{0} \supset K$. Set $s=m_{G}\left(K_{0}^{n}\right)^{-1} \chi_{K_{0}^{n}}$, where $\chi_{K_{0}^{n}}$ denotes the characteristic function of $K_{0}^{n}$. Then $\int s=1$ and $s$ belongs to the center of $L^{1}(G)$. By $(++)$, a routine calculation shows that $\left\|L_{y} s-s\right\|_{1}<\varepsilon, y \in K$.

In the general case we apply the structure theorem for $[F C]^{-}$-groups (see $[9$, Theorem 3D] and [4]). Hence there exists a compact, normal subgroup $H$ such that $G / H=V \times D$, where $V$ is a vector group and $D$ is a discrete $[F C]^{-}$group. Now let $K$ be given as before. We denote the canonical map of $G$ onto $G / H$ by $\pi_{H}$. There exist compact sets $K_{1} \subset V, K_{2} \subset D$ such that $K_{1} \times K_{2}$ $\supset \pi_{H}(K)$. The result for discrete groups combined with [7, p. 112, Chapter 5, 2.1(i)], yields the existence of a central function $\dot{s}$ with $\dot{s} \in L^{1}(G / H), \int \dot{s}=1$, $\dot{s} \geqslant 0$, such that $\left\|L_{\dot{y}} \dot{s}-\dot{s}\right\|_{1}<\varepsilon, \dot{y} \in K_{1} \times K_{2}$. If we put $s=\dot{s} \cdot \pi_{H}$, one can easily see that $s$ has all desired properties.

(b) $\Rightarrow$ (c). Denote by $B$ the set of all pairs $(K, \varepsilon)$ with $K$ compact and $\varepsilon>0$. $B$ is filtering with respect to the relation $\prec$, where $(K, \varepsilon) \prec\left(K^{\prime}, \varepsilon^{\prime}\right)$ means $K \subset K^{\prime}$ and $\varepsilon^{\prime}<\varepsilon$. Now for each $\beta \in B$ there exists an $s_{K, \varepsilon}$ such that $\left\|L_{y} s_{K, \varepsilon}-s_{K, \varepsilon}\right\|_{1}<\varepsilon, y \in K$. Taking $w_{\beta}=s_{K, \varepsilon}$, we see by [7, p. 113, Lemma] that $w_{\beta}$ belongs to the center of $L^{1}(G)$ and that every $f \in L^{0}(G)$ satisfies $\lim _{\beta} f * w_{\beta}=0$.

(c) $\Rightarrow(a)$. Since the center of $L^{1}(G)$ is not trivial $\left(w_{\beta} \neq 0\right)$, there exists a compact, invariant neighbourhood $U$ of the identity (see [5]). Set $g$ $=m_{G}(U)^{-1} \chi_{U}$; it follows that $v_{\beta}=w_{\beta} * g$ is a continuous, central function vanishing at infinity. Furthermore $\int v_{\beta}=1$ and

$(+++) \quad \lim _{\beta}\left\|f * v_{\beta}\right\|_{1}=\lim _{\beta}\left\|f * w_{\beta} * g\right\|_{1} \leqslant \lim _{\beta}\left\|f * w_{\beta}\right\|_{1}=0$.

Let $H$ be the normal subgroup consisting of all elements with precompact 
conjugacy class. $H$ is an open subgroup of $G$ since $U \subset H$. It is known that $f \in L^{1}(G)$ is central iff $f$ satisfies, for all $x \in G, f\left(x y x^{-1}\right)=f(y) \Delta(x)$ a.e. $y \in G$, where the null set may depend on $x$ (see [6, Proposition 1.2]). Since the center of $L^{1}(G)$ is not trivial, $G$ is unimodular [5]. Hence we obtain that $v_{\beta}\left(x y x^{-1}\right)=v_{\beta}(y)$ for all $x, y \in G$, because $v_{\beta}$ is continuous, $\beta \in B$. Therefore the conjugacy class of each $y \in G$ satisfying $v_{\beta}(y) \neq 0$ for any $\beta \in B$ is precompact $\left(v_{\beta}\right.$ is continuous and vanishes at infinity); in particular Supp $v_{\beta}$ $\subset H$. If $G \neq H$, we take an element $a \in G$ such that $a H \cap H=\varnothing$. Choosing any $f_{1} \in L^{1}(G)$ satisfying $f_{1} \geqslant 0, \int f_{1}=1$, Supp $f_{1} \subset H$, we put $f=L_{a} f-f$ and obtain

$$
\operatorname{Supp}\left(v_{\beta} * L_{a} f_{1}\right) \cap \operatorname{Supp}\left(v_{\beta} * f_{1}\right) \subset H \cdot a H \cap H \cdot H=\varnothing .
$$

Hence we get

$$
\left\|f * v_{\beta}\right\|_{1}=\left\|v_{\beta} * L_{a} f_{1}\right\|_{1}+\left\|v_{\beta} * f_{1}\right\|_{1} \geqslant\left(\int v_{\beta}\right) \cdot\left(\int f_{1}\right)=1
$$

in contradiction to $(+++)$. Therefore $G=H$, i.e. $G \in[F C]^{-}$.

Now we are able to prove the theorem.

(i) $\Rightarrow$ (ii). (2) yields the existence of bounded, multiple, approximate, twosided units $\left(v_{\alpha}\right)_{\alpha \in A}$ belonging to the center of $L^{1}(G)$ such that $\int v_{\alpha}=1, \alpha$ $\in A$. Since $G \in[F C]^{-}$, it follows by the proposition above that there exists a net $\left(w_{\beta}\right)_{\beta \in B}$ in the center of $L^{1}(G)$ with $\int w_{\beta}=1$ and $\lim _{\beta} w_{\beta} * f=0, f$ $\in L^{0}(G)$. If we put $u_{\alpha, \beta}=v_{\alpha}-w_{\beta}$, one can easily see that $u_{\alpha, \beta}$ belongs to the center of $L^{0}(G)$, and, moreover, every $f \in L^{0}(G)$ satisfies

$$
\lim _{\alpha, \beta} f * u_{\alpha, \beta}=\lim _{\alpha} f * v_{\alpha}-\lim _{\beta} f * w_{\beta}=f,
$$

i.e. $\left(u_{\alpha, \beta}\right)_{\alpha \in A, \beta \in B}$ is the desired approximate unit.

(ii) $\Rightarrow$ (i). First we show that $G \in[S I N]$. Let $f$ belong to the center of $L^{0}(G)$. We want to show that $f * g=g * f$ holds for every $g \in L^{1}(G)$, i.e. $f$ is in the center of $L^{1}(G)$. We assume that $\int g=1$. Let $\left(v_{\beta}\right)_{\beta \in B}$ be a bounded, multiple, approximate, two-sided unit in $L^{1}(G)$ with $\int v_{\beta}=1, \beta \in B$. Hence we obtain

$$
\begin{aligned}
f * g-g * f= & \lim _{\beta} f *\left(g-v_{\beta}\right)-\lim _{\beta}\left(g-v_{\beta}\right) * f \\
& +\lim _{\beta} f * v_{\beta}-\lim _{\beta} v_{\beta} * f \\
= & 0,
\end{aligned}
$$

because $g-v_{\beta}$ belongs to $L^{0}(G), \beta \in B$. This fact implies that $\left\{u_{\alpha}\right\}$ is contained in the center of $L^{1}(G)$. Next we observe that the operator on $L^{1}(G)$ of left translation by $x$ cannot reduce to the identity on $\left\{u_{\alpha}\right\}$ (otherwise $L_{x} f=f$ holds for any $f \in L^{0}(G)$; a trivial modification of the proof in [5, p. $615]$ shows that this is a contradiction). Now one can imitate step by step the proof of [5]. Therefore $G \in[S I N]$. To show that $G \in[F C]^{-}$it sufficies, by the proposition, to show that there exists a net $\left(w_{\gamma}\right)_{\gamma \in C}$ belonging to the center of $L^{1}(G)$ such that $\int w_{\gamma}=1$ and $\lim _{\gamma} w_{\gamma} * f=0, f \in L^{0}(G)$. If we put $w_{\alpha, \beta}$ $=v_{\beta}-u_{\alpha}$, then $w_{\alpha, \beta}$ belongs to the center of $L^{1}(G)$; furthermore $\int w_{\alpha, \beta}=1$ 
and $\lim _{\alpha, \beta} w_{\alpha, \beta} * f=0, f \in L^{0}(G)$. Hence $G \in[F C]^{-} \cap[S I N]$, i.e. $G$ $\in[F I A]^{-}$.

The methods of our proof admit the following

COROLlary. If $L^{0}(G)$ possesses central approximate units, then $L^{0}(G)$ possesses even bounded, multiple, approximate units consisting of continuous functions with compact support, the norm of the units being bounded by the constant 2 .

\section{BIBLIOGRAPHY}

1. S. Grosser and M. Moskowitz, Compactness conditions in groups, J. Reine Angew. Math. 246 (1971), 1-40. MR 44 \# 1766.

2. On central topological groups, Trans. Amer. Math. Soc. 127 (1967), 317-340. MR 35 \#292.

3. H. Leptin, Zur harmonischen Analyse klassenkompakter Gruppen, Invent. Math. 5 (1968), 249-254. MR 38 \#2257.

4. J. Liukkonen, Dual spaces of groups with precompact conjugacy classes, Trans. Amer. Math. Soc. 180 (1973), 85-108. MR 47 \#6937.

5. R. D. Mosak, Central functions in group algebras, Proc. Amer. Math. Soc. 29 (1971), 613-616. MR 43 \#5323.

6. - The $L^{1}$ - and $C^{*}$-algebra of $[F I A]_{B}^{-}$groups and their representations, Trans. Amer. Math. Soc. 163 (1972), 277-310. MR 45 \# 2096.

7. H. Reiter, Classical harmonic analysis and locally compact groups, Clarendon Press, Oxford, 1968. MR 46 \# 5933.

8. — Sur certain idéaux dans $L^{1}(G)$, C. R. Acad. Sci. Paris Sér. A-B 267 (1968), A882A885. MR 39 \#6025.

9. L. C. Robertson, A note on the structure of Moore groups, Bull. Amer. Math. Soc. 75 (1969), 594-599. MR 39 \#7027.

Mathematics Institute, University of Vienna, A-1090 Vienna, Austria 